\title{
Thermoacoustic waves in pulsed Pirani sensor MEMS. Numerical Investigation
}

\author{
Peter Gospodinov ${ }^{1}$, Dobri Dankov ${ }^{1 *}$, Vladimir Roussinov ${ }^{1}$, Mirona Mironova $^{1}$ \\ ${ }^{1}$ Institute of Mechanics, Bulgarian Academy of Science, Bulgaria
}

\begin{abstract}
The transient heat transfer process is studied in rarefied gas confined between two stationary concentric cylinders. The inner cylinder (filament) is subjected to a periodically heating-cooling cycle. The energy transfer is modeled with continuous model based on Navier-Sockes Fourier equations of motion and energy transfer and with a statistical DSMC model. Numerical results for the temperature, thermodynamic pressure and pressure difference between thermodynamic pressure and radial stress tensor component are obtained for different circular frequencies of heating cooling cycle of filament and for different filament radii. The pressure variation at the end of any local heating stage of heating-cooling cycle is close to the value of equilibrium thermodynamic pressure. The results are applicable in designing the pulsed Pirani sensors.
\end{abstract}

\section{Introduction}

Pirani vacuum gauges usually consist of an electrically heated filament suspended in a cylindrical tube. The filament is kept at a constant temperature. A new gauge is described for measurement of pressures in the range $10^{-4}$ mbars to 5 bars. The gauge uses a conventional Pirani sensor, but pulses the filament temperature and measures filament heating and cooling rates [4]. The physical foundations of this measurement method are considered in [8]. A novel Pirani transmitter which is operated in pulsed mode reveals significant advantages. Its efficiency can be compared to the classical Pirani system. The filament is no longer operated steadily at a constant temperature, but cyclically heated up to a certain temperature threshold by an increasing voltage ramp [10]. Numerical calculations of heat flux in the Pirani sensor were carried out for a wide range of the gas rarefaction based on the kinetic Boltzmann equation. To take into account a non-diffusible gas-surface interaction, the Cercignani-Lampis scattering kernel was used in the boundary condition [11]. Sound waves, propagating through a rarefied gas confined between two coaxial cylinders and induced by an unsteady heating of the inner boundary, are modeled on the basis of a kinetic model to the linearized and non-stationary Boltzmann equation [16].

We study a rarefied monatomic gas between two coaxial, stationary, unconfined cylinders (one dimensional, axis-symmetrical problem). The temperature of the outer cylinder is constant and equal to the gas equilibrium temperature, while the temperature of

\footnotetext{
*Corresponding author: dobri dankov@abv.bg
} 
the inner cylinder varies in time. The inner cylinder (filament) is subjected on periodically heating-cooling cycle.

\section{Continuous Model (NSF) and Numerical Simulation}

The continuous model is based on the Navier-Stokes-Fourier (NSF) equations for compressible fluid, completed with the equations of continuity and energy transport. For details see $[15,17,19]$. The tangential velocity component in the gas flow is $\mathbf{v} \equiv 0$, for $R_{1} \leq r \leq R_{2}$ The coordinates in radial and tangential direction are $(r, \varphi)$.

The following notations are used to describe the rarefied gas flow: $u$ is the velocity component in $r$ directions, $\rho$ is the density and $T$ is the temperature, $P$ is the thermodynamic pressure. The nonzero stress tensor components taking part in the equation of motion [7] can be expressed as.

$$
\begin{gathered}
\tau_{r r}=-\mu\left(2 \frac{\partial u}{\partial r}-\frac{2}{3} \frac{1}{r} \frac{\partial}{\partial r}(r u)\right) \\
\tau_{\varphi \varphi}=\mu\left[\frac{2}{3}\left(\frac{1}{r} \frac{\partial}{\partial r}(r u)-2\left(\frac{u}{r}\right)\right)\right]
\end{gathered}
$$

The dissipation function $\Phi$ [7] in the equation of energy transport is

$$
\Phi=\frac{4}{3} \mu\left[\left(\frac{\partial u}{\partial r}\right)^{2}-\frac{\partial u}{\partial r} \frac{u}{r}+\left(\frac{u}{r}\right)^{2}\right]
$$

For a perfect monatomic gas, the viscosity and the heat transfer coefficients read as $[1$, $2,3,6]$ :

$$
\begin{aligned}
& \mu=\mu(T)=C_{\mu} \rho_{0} l_{0} V_{0} \sqrt{T}, C_{\mu}=\frac{5}{16} \sqrt{\pi} \\
& \lambda=\lambda(T)=C_{\lambda} \rho_{0} l_{0} V_{0} \sqrt{T}, C_{\lambda}=\frac{15}{32} \sqrt{\pi}
\end{aligned}
$$

The NSF model is completed with the equation of state for ideal gas:

$$
P=\rho R T
$$

The governing equations are normalized by using the following scales: for density $\rho_{0}=m n_{0}$ ( $m$ is the molecular mass, $n_{0}-$ the average number density), for velocity $V_{0}=\sqrt{2 R T_{0}}-R$ is the gas constant, for length - the distance between the cylinders $L=R_{2}-R_{1}$, for time $t_{0}=L / V_{0}$, for temperature $T_{0}-$ the wall temperature of both cylinders. The Knudsen number is $\mathrm{Kn}=l_{0} / L$, where the mean free path is $l_{o}$ and $\gamma=c_{P} / c_{V}=5 / 3 \quad\left(c_{\mathrm{P}}\right.$ and $c_{\mathrm{V}}$. are the heat capacities at constant pressure and constant volume respectively). In this way, the characteristic number Kn and the constants $C_{\mu}$ and $C_{\lambda}$ take part in the dimensionless model.

For the problem formulated first-order slip boundary conditions are imposed at both walls which can be written directly in dimensionless form as follows [19]:

$$
u=0
$$




$$
T \pm \varsigma_{T} \operatorname{Kn}_{\text {local }} \frac{\partial T}{\partial r}=\bar{T}_{i}
$$

at $r=R_{i},: i=1$, 2. In Eqs. (7)-(8) $V_{i}=v_{i} / V_{0}$ is the dimensionless wall velocity, ( $v_{i}, i=1,2$ is the dimensional wall velocity). For diffuse scattering we have used the temperature jump coefficients $\zeta_{\mathrm{T}}=2.1904$ calculated, respectively, from the kinetic Bhatnagar-Gross-Krook (BGK) equation [2, 14],

The dimensionless wall pulsating temperature on the filament $\bar{T}_{1}$ depends on the dimensionless time $t$ harmonically:

For

$$
2 \pi k \leq \varpi t \leq 2 \pi(k+1 / 2), \text { and }
$$

$2 \pi(k+1) \leq \varpi t \leq 2 \pi(k+1+1 / 2), k=1,2,3, \ldots \ldots$

$$
\bar{T}_{1}=T_{1,0}[1+A \sin \mid(\varpi t)]
$$

where $T_{1,0}$ is the inner cylinder (filament) wall temperature value for $\varpi t=\pi / 2+k \pi$, and $A=0.1$ is the oscillations amplitude. The condition (8)-(9) describes the filament heating stage from the heating-cooling cycle.

For $2 \pi(k+1 / 2) \leq t \leq 2 \pi(k+1)$ instead (8) is applied the adiabatic condition (10), corresponding to the cooling stages of the heating-cooling cycle.

$$
\frac{\partial T}{\partial r}=0, r=R_{1}
$$

$T_{2}=1$ is the outer cylinder wall constant temperature.

The boundary conditions are modeled by using the local Knudsen number $\mathrm{Kn}_{\text {local. }}$ [20]

$$
\mathrm{Kn}_{\text {local }}=\frac{l}{L}=\left(L \sqrt{2} \pi \sigma^{2} \rho n_{0}\right)=\frac{\mathrm{Kn}}{\rho}
$$

In (11) with $l$ is denoted the local mean free path, $\sigma$ - molecular diameter and $\rho$ is the dimensionless density.

Following $[9,12,15]$ we introduce a Stokes number for temperature oscillations $\beta=\sqrt{\frac{\omega L^{2}}{v}}$, where $v$ is kinematic viscosity and $\omega-$ temperature circular frequency. The relation between dimensionless temperature frequency $\bar{\omega}$ and Stokes number $\beta$ can be written, using eq. Eq.(4).

$$
\bar{\omega}=C_{\mu} \mathrm{Kn} \beta^{2}
$$

The dimensionless value of thermodynamic pressure $P_{0}$ corresponding to initial equilibrium state can be calculated as:

$$
P_{0}=\frac{1}{2} \rho_{0} T_{0}=\frac{1}{2}
$$

In Eq.(13) $\rho_{0}$ and $T_{0}$ are the equilibrium dimensionless density and temperature.

The heating-cooling cycle is the reason why the thermoacoustic waves arise in the gas media. In fact, the measured pressure is the pressure difference defined as [16]:

$$
\Pi=P-\tau_{r r}
$$




\section{Direct Simulation Monte Carlo (DSMC) Method}

The gas considered is simulated as a stochastic system of $N$ particles [5]. All quantities used are non-dimensional, so that the mean free path at equilibrium is equal to 1 . The basic steps of simulation are as follows:

A. The time interval $[0 ; \hat{t}]$ over which the solution is found, is subdivided into subintervals with step $\Delta t$.

B. The space domain is subdivided into cells with sides $\Delta z, \Delta r$.

C. Gas molecules are simulated in gap $\mathrm{G}$ using a stochastic system of $N$ points (particles), having position $z_{i}(t), r_{i}(t)$ and velocities $\left(\xi_{z}^{i}(t), \xi_{r}^{i}(t), \xi_{\varphi}^{i}(t)\right)$,

D. $N_{m}$ particles are located in the $m$-th cell at any given time. This number varies during the computer simulation by the following two stages:

Stage 1. Binary collisions in each cell are calculated, whereas particles do not move. Collision modeling is realized using Bird's scheme "no time counter".

Stage 2. Particles move with new initial velocities, acquired after collisions and no external forces act on particles. No collisions are accounted for at this stage.

E. Stage 1 and Stage 2 are repeated until $t=\hat{t}$.

F. Flow macro-characteristics (density, velocity, temperature) are calculated as timeaveraged.

G. Boundary conditions are: diffusive at the outer cylinder wall; diffusive or adiabatic at the inner cylinder wall depending on the time $t$; periodic along axis $O z$.

We denote the particle velocity before collision with wall with $\left(\xi_{z}, \xi_{r}, \xi_{\varphi}\right)$ and after with $\left(\xi_{z}^{*}, \xi_{r}^{*}, \xi_{\varphi}^{*}\right)$, respectively the velocity magnitude with $c=\sqrt{\xi_{z}^{2}+\xi_{r}^{2}+\xi_{\varphi}^{2}}$ and $c^{*}=\sqrt{\xi_{z}^{* 2}+\xi_{r}^{* 2}+\xi_{\varphi}^{* 2}}$. This gas-surface interaction model can be described by keeping the particle velocity magnitude invariant and the velocity direction of the reflected particle set based on isotropic scattering boundary conditions in the half unite sphere. We discuss this problem first in the spherical coordinate system $\left(c, \alpha_{1}, \alpha_{2}\right)\left(\alpha_{1} \in\left[0, \frac{\pi}{2}\right], \alpha_{2} \in[0,2 \pi]\right)$ $[13,18]$. The three velocity components on the inner cylinder for adiabatic case reflected by:

$$
\begin{aligned}
& \xi_{r}^{*}=c \cdot \cos \alpha_{1} \\
& \xi_{z}^{*}=c \cdot \sin \alpha_{1} \cdot \cos \alpha_{2} \\
& \xi_{\varphi}^{*}=c \cdot \sin \alpha_{1} \cdot \sin \alpha_{2}
\end{aligned}
$$

and for diffusion case reflected by:

$$
\begin{aligned}
& \xi_{r}^{*}=\sqrt{\frac{2 k_{B} \bar{T}_{1}}{m}} \cdot \cos \alpha_{1} \\
& \xi_{z}^{*}=\sqrt{\frac{2 k_{B} \bar{T}_{1}}{m}} \cdot \sin \alpha_{1} \cdot \cos \alpha_{2} \\
& \xi_{\varphi}^{*}=\sqrt{\frac{2 k_{B} \bar{T}_{1}}{m}} \cdot \sin \alpha_{1} \cdot \sin \alpha_{2}
\end{aligned}
$$


The difference between diffusion and adiabatic reflection is only the coefficient. At the diffusion reflection the coefficient $\sqrt{\frac{2 k_{B} \bar{T}_{1}}{m}}$ depends on the wall temperature $\bar{T}_{1}$ and the particle mass $m$, while at the adiabatic reflection the coefficient $c$ is the particle velocity magnitude before collision on the particle with the wall.

The temperature on the filament $\bar{T}_{1}$ depends on the dimensionless time $t$ harmonically for $2 \pi k \leq \varpi t \leq 2 \pi(k+1 / 2)$, and $2 \pi(k+1) \leq \varpi t \leq 2 \pi(k+1+1 / 2), k=1,2,3, \ldots \ldots$. The boundary condition is modeled by (16). The adiabatic condition (16) is applies for $2 \pi(k+1 / 2) \leq t \leq 2 \pi(k+1)$ instead (15), corresponding to the cooling stage from the heating-cooling cycle.

\section{Numerical results and discussion}

The numerical solution of the NSF and DSMC models in detail is described in $[15,17$, 19].

We study three main cases with different frequency $-\beta=2, \beta=4, \beta=8$ In numerical calculation is used the Knudsen number $\mathrm{Kn}=0.02$, inner cylinder radius $R_{1}=0.05,0.005,0.001$ and outer cylinder radius respectively $R_{2}=1.05,1.005,1.001$. Numerical results for filament temperature (Fig.1, 2 ,3 and 5), pressure $P$ (Fig.4) and pressure difference $\Pi$ (Fig.1, 2, 3, 4) are presented. The values of the macrocharacteristics for a fixed dimensionless time value are obtained as a mean value in the radial direction.

The filament temperature variation $0 \leq t \leq 300$ is shown on the Fig.1, 2 and 3. At the lowest frequency, the dimensionless time to reach sustained oscillations for filament temperature and macrocharacteristics is less than the half period - Fig.1, 2, 3, while at higher frequencies this time is in the order of 30 (Fig.5 left) and 50 (Fig.5 right).

The filament temperature pulsation generates thermoacoustic waves in the direction of the radius that are reflected between the two cylinder walls. This causes the deviation of the gas pressure from its equilibrium value. The pressure difference $\Pi$ is the actual measured value with the gauge [16]. The stress tensor variation limits are negligible and the difference between thermodynamic pressure and pressure difference is negligible (Fig.4). The pressure difference local extreme coincides at the time with the local extreme temperature after reaching the sustained oscillations. No phase difference was observed for the studied cases. It can be concluded that, while maintaining the amplitude of filament temperature, the pressure variation limits are narrowed at increasing the pulses' forced frequency.

The filament radius decreasing decreases the amplitudes of filament temperature and pressure differential oscillations.

The local maxima of sustained oscillations of pressure difference are close to the value of the equilibrium thermodynamic pressure when decreasing the filament radius. (Fig. 6) 

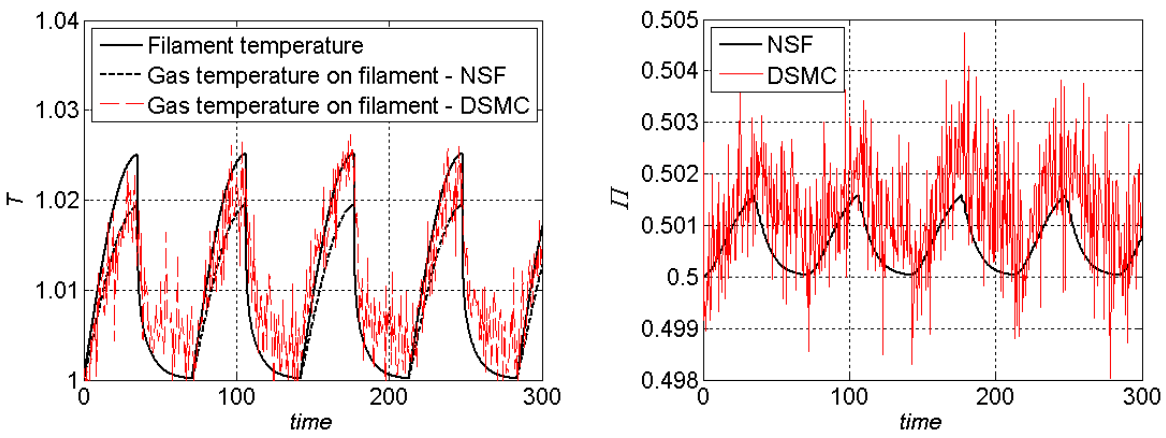

Fig. 1. The temperature and the pressure difference variation at $\beta=2$ and $R_{1}=0.05$
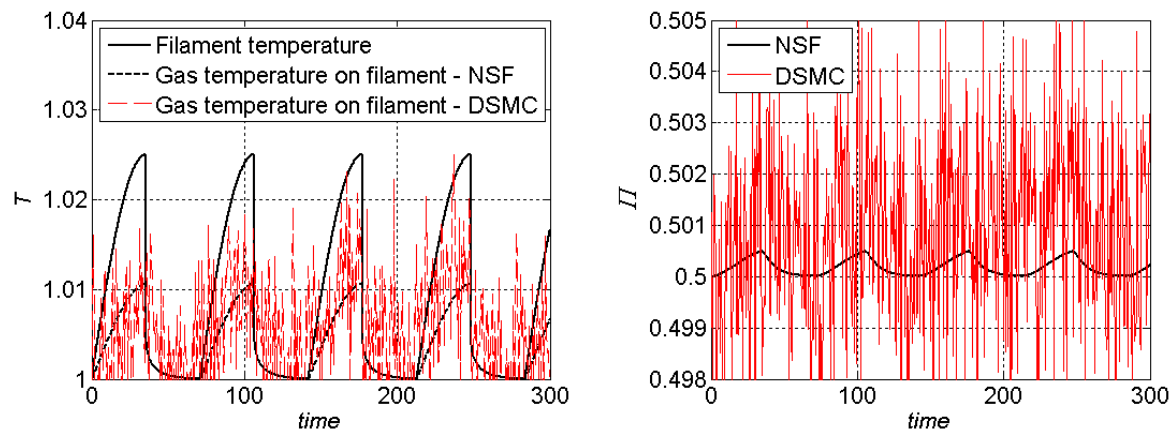

Fig. 2. The temperature and the pressure difference variation at $\beta=2$ and $R_{1}=0.005$
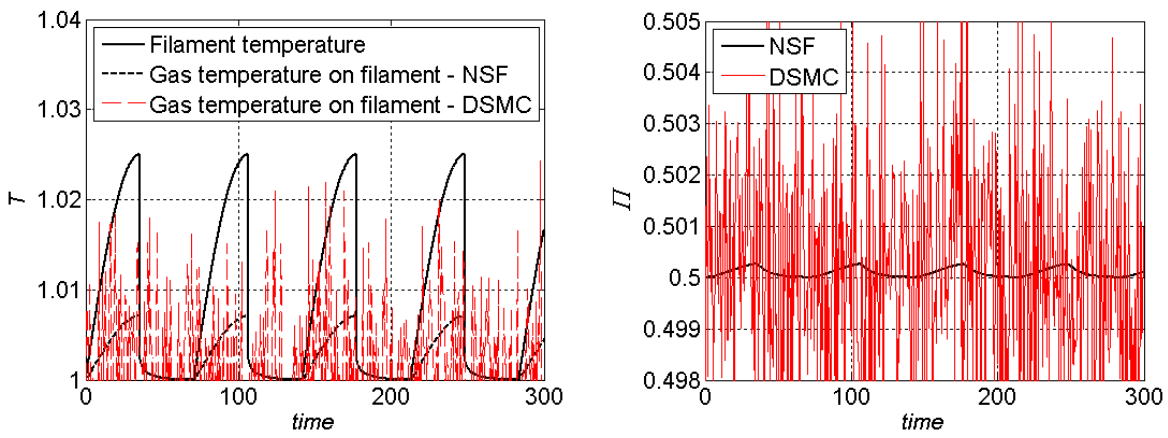

Fig. 3. The temperature and the pressure difference variation at $\beta=2$ and $R_{1}=0.05$ 

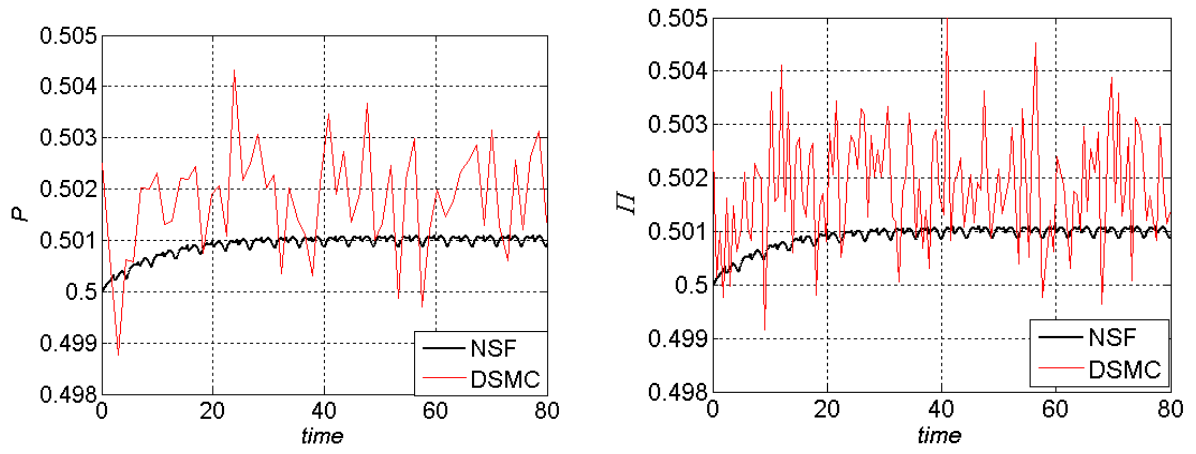

Fig. 4. The temperature and the pressure difference variation at $\beta=2$ and $R_{1}=0.05$
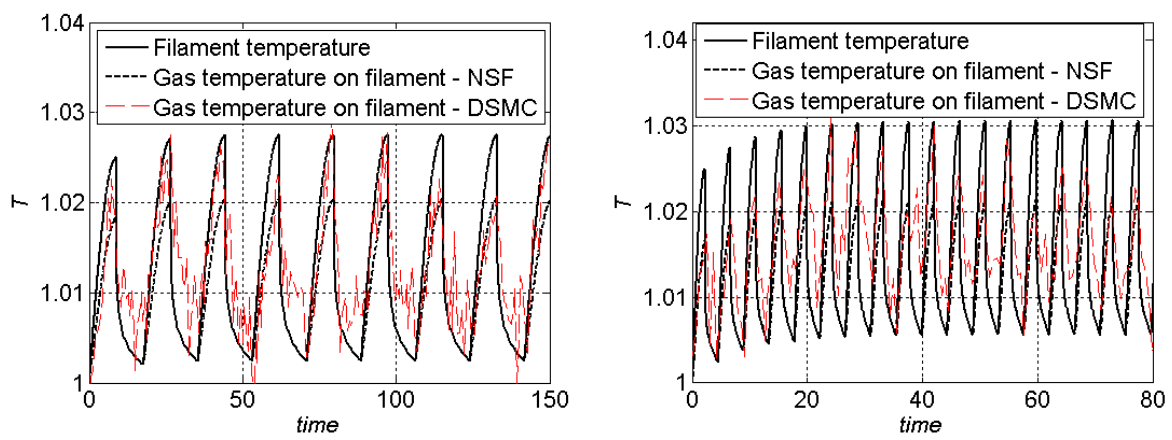

Fig. 5. The fiber temperature variation at $\beta=4$ (left figure) and $\beta=8$ (right figure).

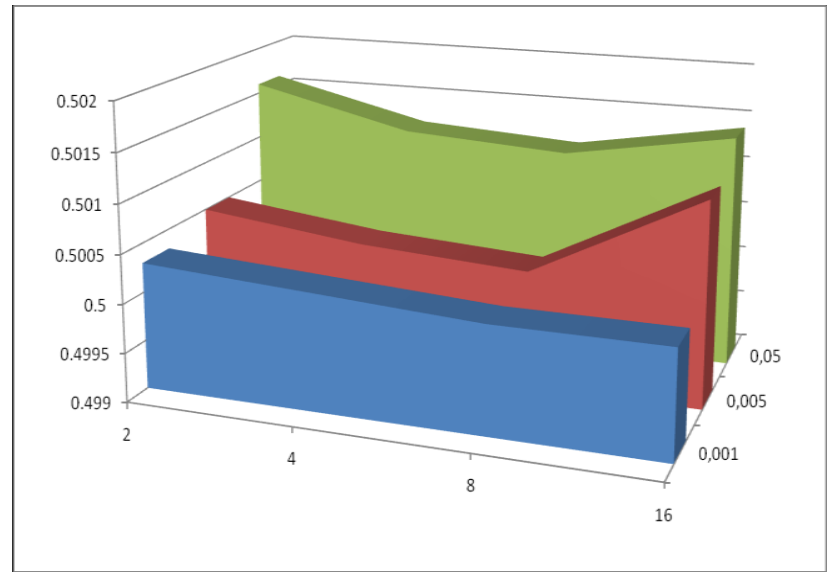

Fig. 5. The local maxima of sustained oscillations of pressure difference.

\section{Conclusion}

The differences in numerical results between the two models appear visually large but as values are below $0.5 \%$. This means that there were no significant differences in numerical results obtained by two methods - NSF and DSMC. 
Numerically is analyzed the operation of the MEMS pulsating Pirani sensor. The actual read pressure (pressure difference) variation is studied. At the heating stage end of heatingcooling cycle the pressure difference is close to the value of equilibrium thermodynamic pressure.

The results can be used to analyze the pulsating Pirani sensor sensitivity, i.e. the time to reach sustained oscillations as well as to adjust the pressure reading to the actual value.

Acknowledgment: V. Roussinov would like to thank for the financial support provided by the NSF of Bulgaria under Grant No DN 02/7-2016.

\section{References}

1. S. Chapman, T. Cowling, The Mathematical Theory of Non-Uniform Gases (Cambridge University Press, Cambridge, 1952).

2. S. Albertoni, C. Cercignani, L. Gotusso, Phys. Fluids 6, 993-996 (1963)

3. C. Cercignani, F. Sernagiotto, Physics of Fluids 10, 1200-1204 (1967)

4. M. Cole, Vacuum 38(8-10), 897-899 (1988)

5. G. A. Bird, Molecular Gas Dynamics and the Direct Simulation of Gas Flows (Oxford University Press, Oxford, 1994).

6. C. Cercignani, Rarefied Gas Dynamics, From Basic Concepts to Actual Calculations (Cambridge University Press, Cambridge, 2000).

7. R. Bird, W. Stewart, and E. Lightfoot, Transport Phenomena, 2nd edn (John Wiley, New York, 2002).

8. W. Jitschin, S. Ludwig, Vakuum in Forschung und Praxis 16, 23-29 (2004)

9. J. Park, P. Bahukudumbi, A. Beskok, Phys. Fluids 16, 317 (2004)

10. H. Ploechinger, F. Salzberger, Pulsed Pirani vacuum gauge (2005): http://www. researchgate.net/publication/260123890.

11. F. Sharipov, G. Bertoldo, Numerical modelling of Pirani sensor, in XVIII IMEKO World Congress, Metrology for a Sustainable Development, Rio de Janeiro, Brazil, September, 17-22, 2006.

12. D. Emerson, Gu Xiao-Jun, S. Stefanov, S. Yuhong, W. Barber, Phys. Fluids 19, 107105 (2007)

13. P. Tzeng, W. Chou, C. Liu, W. Li, Journal of Aeronautics, Astronautics and Aviation, Series A 43(3), 147-158 (2011)

14. F. Sharipov, J. Phys. Chem. Ref. Data 40, 023101 (2011)

15. P. Gospodinov, V. Roussinov, S. Stefanov, European Journal of Mechanics B/Fluids 33, 68-77 (2012)

16. D. Kalempa, F. Sharipov, Vacuum 109, 326-332 (2014)

17. P. Gospodinov, V. Rousinov, M. Mironova, Cylindrical nonisothermal oscillatory Couette gas flow in the slip regime: Wall shear stress and energy transfer, numerical investigation, Intellectual Archive Bulletin, Feb. 19, pp. 2-19 (2014)

18. A. Mohammadzadeh, A. Rana, H. Struchtrup, International Journal of Thermal Sciences 101, 9-23 (2016)

19. P. Gospodinov, D. Dankov, and V. Roussinov, AIP Conference Proceedings 1773, paper 080001 (2016)

20. P. Gospodinov, D. Dankov, V. Roussinov, S. Stefanov, AIP Conference Proceedings 1404, 451-456 (2011) 